\title{
Impacto epidemiológico y económico de la vacunación contra el tétanos en adultos de Colombia
}

\author{
Nelson Alvis, ${ }^{1}$ Fernando De La Hoz, ${ }^{2}$ Oscar Gamboa, ${ }^{2}$ \\ Natalia Cediel, ${ }^{2}$ Alejandro Rico, ${ }^{2}$ Ángel Paternina ${ }^{1}$ y Luis Alvis $^{1}$
}

Forma de citar $\begin{aligned} & \text { Alvis N, De La Hoz F, Gamboa O, Cediel N, Rico A, Paternina A, et al. Impacto epidemiológico y } \\ & \text { económico de la vacunación contra el tétanos en adultos de Colombia. Rev Panam Salud Publica. }\end{aligned}$ económico de la vac

RESUMEN Objetivo. Evaluar el potencial impacto epidemiológico y económico de la vacunación antitetánica en la población colombiana mayor de 15 años de edad, con dosis de refuerzo cada 10 años. Métodos. Se realizó un análisis de costo-efectividad de la vacunación con refuerzo cada 10 años contra el tétanos en Colombia, comparándola con la actual estrategia $(2,4,6,18$ y 60 meses). La carga de enfermedad se estimó con base en tres fuentes oficiales de datos. Se realizó un modelo de Markov desde la perspectiva del tercer pagador. El horizonte temporal fue el tiempo de vida de una persona. Se realizaron análisis de sensibilidad determinístico y probabilístico.

Resultados. En Colombia se notifican anualmente entre 30 y 48 casos de tétanos que ocasionan entre 9,6 y 10,1 muertes. Si bien la vacunación con refuerzo resultó costo-efectiva para toda la población (el costo por año de vida ajustado por discapacidad [AVAD] evitado fue de US\$ 11314 en toda la población), al discriminar los resultados por género no sería costoefectiva en las mujeres (el costo por AVAD evitado en hombres fue de US\$ 4 903, y en mujeres de US\$22 332).

Conclusiones. Este es el primer estudio que evalúa la costo- efectividad de una dosis de refuerzo cada 10 años de la vacuna contra el tétanos en un país en desarrollo. La aplicación de esta medida sería costo-efectiva en Colombia, especialmente para los hombres. Las diferencias en los resultados por género obliga a que cualquier decisión de implementación deba tener en cuenta la vacunación actual en mujeres en edad fértil.

Palabras clave Toxoide tetánico; economía de la salud; salud materno-infantil; Colombia.

El tétanos es una enfermedad infecciosa aguda, altamente letal, causada por una exotoxina producida por la bacteria Clostridium tetani. Aunque tiene una distribución mundial, se observa con mayor frecuencia en zonas rurales, cálidas y hú-

\footnotetext{
1 Universidad de Cartagena, Cartagena, Colombia. La correspondencia se debe dirigir a Nelson Alvis. Correo electrónico: nalvis@yahoo.com

2 Universidad Nacional de Colombia, Facultad de Medicina, Bogotá, Colombia.
}

medas, circunstancias que favorecen la supervivencia prolongada de las esporas $(1,2)$.

La incidencia del tétanos se relaciona estrechamente con el estado de inmunización de la población, por lo cual en los países donde los programas de vacunación no son adecuados se registran tasas aproximadas de 20 casos por cada 100000 habitantes al año, mientras que donde la inmunización responde a los niveles requeridos dicha tasa es apenas de $0,1 / 100$ 000. Su mortalidad en tanto varía según la gravedad, oscilando entre $10 \%$ y $60 \%$ en el tétanos generalizado y entre $70 \%$ y $90 \%$ en el neonatal (3).

El tétanos se previene con el toxoide tetánico —en preparación única o combinado con el toxoide diftérico- y con la vacuna contra difteria, tétanos y pertusis celular (DTP) celular o acelular (DTP y $\mathrm{DTaP})(1,2)$. Si bien la eficacia de la va- 
cuna contra el tétanos todavía no se ha comprobado en estudios clínicos, un esquema completo de vacunación (tres dosis de la vacuna) tendría una efectividad de $100 \%$ y se mantendría al menos por 10 años $(2,4)$. En Colombia la vacunación contra el tétanos en niños incluye tres dosis de DTP (como vacuna pentavalente) a los 2, 4 y 6 meses de edad y dos dosis adicionales de DTP a los 18 meses y 5 años de edad. En las mujeres en edad fértil (MEF) se utiliza toxoide tetánico diftérico de adulto (Td). La vacunación en este grupo de población es opcional y se realiza en un esquema de cinco dosis: primera aplicación (cero) y luego 1, 6, 18 y 30 meses después (5).

En los países desarrollados, donde gracias a la vacunación prácticamente han logrado erradicar el tétanos neonatal y accidental, la enfermedad casi se limita a adultos que no reciben refuerzos con toxoide cada decenio y quedan más desprotegidos (6-9). Los principales grupos en riesgo son los hombres, por estar más predispuestos a lesiones, los diabéticos y los consumidores de drogas intravenosas (10-12). En contraste, la enfermedad sigue siendo un problema importante en los países en desarrollo, especialmente el tétanos neonatal $(10,13)$-los países de África y el sureste asiático y Haití son los lugares con mayor frecuencia de muertes $(6,10,14-15)$.

La vacunación tiene como objetivos eliminar el tétanos materno y el neonatal, y prevenir la enfermedad en adultos manteniendo altas coberturas de esquemas con DTP, seguidos por los refuerzos pertinentes $(16,17)$. En Colombia, pese a que ya debería haberse eliminado el tétanos neonatal (TNN), aún se reportan tasas de 1,0 a 7,2 casos por 1000 (18). Adicionalmente, la frecuencia de tétanos accidental es 5 a 10 veces superior a la de TNN $(7,9,19-23)$. Las madres de los bebés afectados con TNN son a menudo multíparas y analfabetas, viven en regiones rurales remotas y en condiciones de pobreza, no reciben atención prenatal y no han sido vacunadas $(16,17)$.

Desde 1997 la incidencia de tétanos accidental en mayores de un año en Colombia se ha mantenido en alrededor de 0,4 casos por 100000 habitantes, siendo más frecuente en niños de 1 a 14 años, con una tasa global de letalidad de $32 \%$ (18). En 2007 el Ministerio de la Protección Social (MPS) planteó la situación de esta enfermedad al Comité Nacional de Prácticas en Inmunización, el cual reco-

FIGURA 1. Modelo de Markov para valorar la costo-efectividad de la vacunación contra el tétanos en adultos

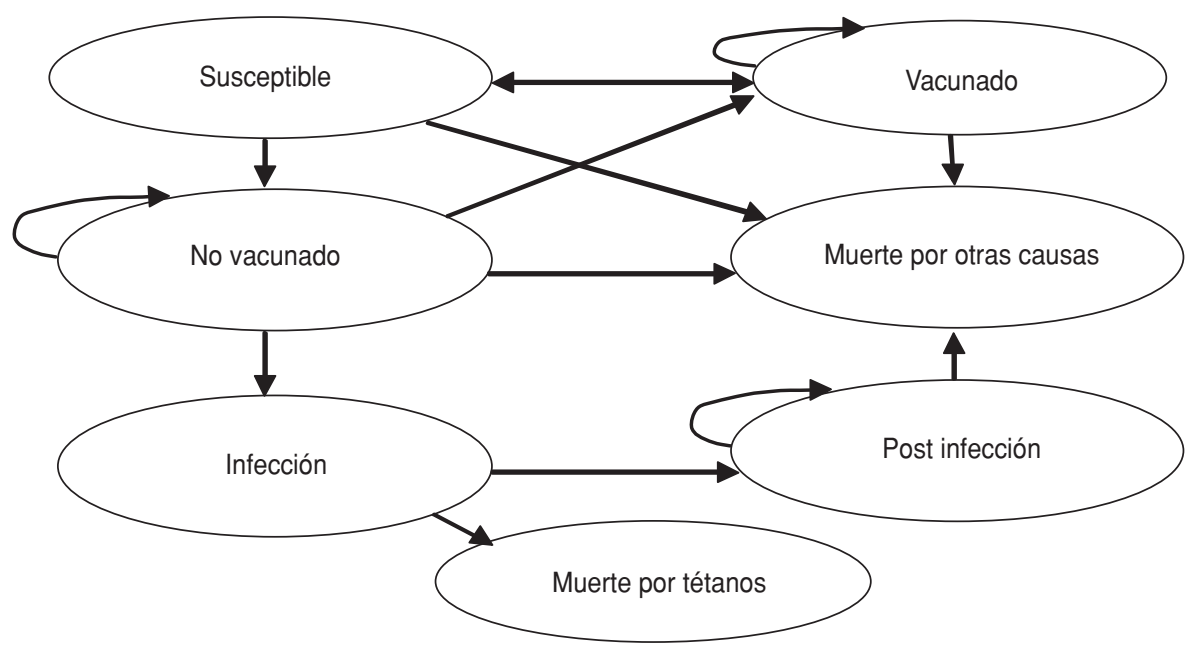

Fuente: elaboración de los autores.

Nota: las flechas indican las transiciones entre los diferentes estados del modelo.

mendó un refuerzo de vacunación contra el tétanos cada 10 años a partir de los 25 años o por lo menos a los 25,35 y 45 años de edad.

El presente trabajo tiene como objetivo evaluar el potencial impacto epidemiológico y económico de la vacunación antitetánica en la población colombiana mayor de 15 años de edad, con dosis de refuerzo cada 10 años.

\section{MATERIALES Y MÉTODOS}

Se combinaron un estudio de carga de enfermedad del tétanos en Colombia y una evaluación económica de los posibles impactos a lograr con la introducción de la vacuna contra el tétanos en población adulta. Para la evaluación económica se construyó un modelo de Markov, en el que se analizaron dos alternativas: i) vacunación con el esquema actual y ii) vacunación según el esquema actual más refuerzo cada 10 años desde los 15 años de edad (figura 1).

\section{Estrategia de vacunación}

La estrategia de vacunación actual de Colombia contra el tétanos contempla la aplicación de DTP a los 2, 4 y 6 meses, y dosis de refuerzo a los 18 meses y 5 años (5). Se supuso una cobertura de $92 \%$ para el escenario base de la vacunación por tétanos, mientras que para los análisis se supuso que la eficacia de la vacuna era de $100 \%$. El costo por vacuna se obtuvo de acuerdo a datos del MPS, estableciéndose en el escenario base en US\$1,4, valor que incluye costos de manejo y envío, y gastos extras del sistema (cuadro 1).

\section{Estimación de la carga de enfermedad}

Para determinar la carga de enfermedad por tétanos en adultos se realizó una búsqueda sistemática de información en las bases de datos de MEDLINE, LILACS y SciELO, con los siguientes términos de búsqueda: Clostridium tetani, tetanos, Cost of Illness, burden of disease, Prevalence, Incidence, Hospitalization, mortality, Mortality, epidemiology, ((Americas NOT North America) OR Mexico), (Age Groups NOT Infants), o sus términos equivalentes en español, en diferentes combinaciones, para localizar artículos con datos de ocurrencia de los eventos desde 1990. De igual forma se realizó una búsqueda en estas mismas bases de datos para determinar la eficacia de la vacuna.

Por otro lado, se indagó en tres fuentes de datos nacionales: i) el Sistema de Vigilancia Epidemiológica (SIVIGILA); ${ }^{3}$ ii) los registros individuales de prestación de servicios de salud (RIPS), que aportaron información con base en la Clasificación Internacional de Enfermedades (CIE) versiones 9 y 10 acerca de

Sistema de información que reporta los casos de mortalidad y morbilidad de enfermedades bajo vigilancia en salud pública. Solo incluye los casos notificados por las unidades primarias generadoras de datos (UPGD) en el territorio nacional y no hace diferencia entre hospitalizaciones y casos. 
CUADRO 1. Parámetros usados en los análisis de sensibilidad determinístico y probabilístico para evaluar el impacto epidemiológico y económico de la vacuna antitetánica en adultos, Colombia, 2009

\begin{tabular}{|c|c|c|c|c|c|c|}
\hline \multirow[b]{4}{*}{ Variable } & \multicolumn{5}{|c|}{ Análisis } & \multirow[b]{4}{*}{ Fuente } \\
\hline & \multicolumn{3}{|c|}{ Determinístico } & & & \\
\hline & \multirow[b]{2}{*}{ Base } & \multicolumn{2}{|c|}{ Rango } & \multicolumn{2}{|c|}{ Probabilístico } & \\
\hline & & Mínimo & Máximo & Distribución & Parámetro & \\
\hline Costo por episodio de tétanos (US\$) & 22856,30 & 18417,80 & 27294,80 & LogNormal & $\begin{array}{c}\mu=17,62 \\
\sigma=0,19804\end{array}$ & Presente estudio \\
\hline Costo vacuna (US\$) & 1,40 & 0,74 & 2,90 & $N A^{a}$ & NA & Ministerio de la Protección Social \\
\hline Riesgo de muerte por tétanos (letalidad) & 0,35 & 0,20 & 0,50 & $\beta$ & $\begin{array}{c}\alpha=16,25 \\
\beta=30,17857\end{array}$ & $\begin{array}{l}\text { Gouveia et al. (24) } \\
\text { Trujillo M (25) }\end{array}$ \\
\hline Cobertura de la vacunación & 0,92 & 0,70 & 0,95 & NA & NA & Supuesto ${ }^{b}$ \\
\hline Peso de la discapacidad de un episodio de tétanos & 0,64 & 0,50 & 0,78 & NA & NA & $\begin{array}{l}\text { Organización Mundial del la } \\
\text { Salud (26) }\end{array}$ \\
\hline
\end{tabular}

Fuente: elaboración de los autores a partir de las referencias mencionadas.

a NA: los datos no son aplicables.

b Se supuso que el refuerzo cada 10 años de la vacunación por tétanos en Colombia fue de $92 \%$.

hospitalizaciones por TNN (A33X), tétanos obstétrico (A34X) y otros tétanos (A35X), por año, por grupo de edad y por departamento de 2002 a 2007, y iii) el Departamento Administrativo Nacional de Estadísticas (DANE), del que se extrajeron las estadísticas de mortalidad entre 1997 y 2005.

Los datos sobre morbilidad fueron tomados del SIVIGILA y los de hospitalización y consultas, de los RIPS, en tanto que para el análisis de la mortalidad se usaron tanto el SIVIGILA como el DANE, que es la fuente oficial para este indicador en el país.

\section{Estimación del impacto económico}

Para estimar la costo-efectividad del refuerzo de vacunación contra el tétanos se construyó un modelo de Markov, compuesto por seis estados: susceptible, vacunado, no vacunado, infección, post infección y muerte, definidos de acuerdo al riesgo de desarrollar infección por tétanos (figura 1). Solamente en el estado "no vacunado" se puede desarrollar la infección; un individuo vacunado puede pasar al estado no vacunado si después de 10 años de haberse aplicado el esquema inicial no se vacuna nuevamente.

El modelo utilizado aquí parte de una cohorte de recién nacidos a quienes se les aplica el esquema habitual de vacunación y se siguen en ciclos anuales hasta que mueran por tétanos u otra causa. Los individuos que sufren un episodio de tétanos pueden morir por esta causa o pasar al estado post infección, donde el riesgo de desarrollar un nuevo episodio se su-

CUADRO 2. Riesgo de infección por tétanos estratificado por grupos de edad y género, Colombia, 2009

\begin{tabular}{|c|c|c|c|c|c|c|}
\hline \multirow{2}{*}{$\begin{array}{l}\text { Grupo de } \\
\text { edad (años) }\end{array}$} & \multicolumn{3}{|c|}{ Hombres } & \multicolumn{3}{|c|}{ Mujeres } \\
\hline & Base & Límite inferior & Límite superior & Base & Límite inferior & Límite superior \\
\hline$<5$ & $1,10(\mathrm{E}-06)$ & $7,50(\mathrm{E}-07)$ & $1,90(\mathrm{E}-06)$ & 0 & 0 & 0 \\
\hline 5 a 9 & $1,50(\mathrm{E}-06)$ & $1,10(\mathrm{E}-06)$ & $2,70(\mathrm{E}-06)$ & $1,10(\mathrm{E}-06)$ & 7,60 (E-07) & $1,90(\mathrm{E}-06)$ \\
\hline 10 a 14 & 2,30 (E-06) & $1,60(E-06)$ & $4,10(\mathrm{E}-06)$ & $1,10(\mathrm{E}-06)$ & 7,90 (E-07) & 2,00 (E-06) \\
\hline 15 a 19 & $1,70(\mathrm{E}-06)$ & 1,20 (E-06) & 3,00 (E-06) & 3,00 (E-07) & 2,10 (E-07) & 5,30 (E-07) \\
\hline 20 a 24 & $6,50(\mathrm{E}-07)$ & 4,60 (E-07) & $1,10(\mathrm{E}-06)$ & 0 & 0 & 0 \\
\hline 25 a 29 & 3,70 (E-07) & 2,60 (E-07) & 6,40 (E-07) & 0 & 0 & 0 \\
\hline 30 a 34 & $1,30(E-06)$ & 8,80 (E-07) & $2,20(\mathrm{E}-06)$ & $3,80(E-07)$ & 2,70 (E-07) & 6,70 (E-07) \\
\hline 35 a 39 & $2,50(\mathrm{E}-06)$ & $1,80(E-06)$ & $4,40(\mathrm{E}-06)$ & 0 & 0 & 0 \\
\hline 40 a 44 & 8,90 (E-07) & 6,30 (E-07) & $1,60(\mathrm{E}-06)$ & $4,10(\mathrm{E}-07)$ & 2,90 (E-07) & 7,20 (E-07) \\
\hline 45 a 49 & $3,80(E-06)$ & 2,70 (E-06) & $6,70(\mathrm{E}-06)$ & 0 & 0 & 0 \\
\hline 50 a 54 & $2,70(E-06)$ & $1,90(\mathrm{E}-06)$ & $4,80(\mathrm{E}-06)$ & 2,50 (E-06) & 1,80 (E-06) & 4,40 (E-06) \\
\hline 55 a 59 & $1,10(\mathrm{E}-05)$ & 8,00 (E-06) & $2,00(\mathrm{E}-05)$ & $4,80(\mathrm{E}-06)$ & 3,30 (E-06) & $8,30(\mathrm{E}-06)$ \\
\hline 60 a 64 & $1,40(E-05)$ & 9,50 (E-06) & 2,40 (E-05) & 6,20 (E-06) & 4,40 (E-06) & 1,10 (E-05) \\
\hline 65 a 69 & $1,50(E-05)$ & $1,10(\mathrm{E}-05)$ & 2,60 (E-05) & 6,00 (E-06) & 4,20 (E-06) & 1,10 (E-05) \\
\hline 70 a 74 & $3,90(E-05)$ & 2,70 (E-05) & 6,80 (E-05) & 7,90 (E-06) & 5,50 (E-06) & 1,40 (E-05) \\
\hline 75 a 79 & $7,70(\mathrm{E}-06)$ & 5,40 (E-06) & $1,30(\mathrm{E}-05)$ & $1,70(E-05)$ & $1,20(\mathrm{E}-05)$ & 3,00 (E-05) \\
\hline$\geq 80$ & $2,30(\mathrm{E}-05)$ & $1,60(\mathrm{E}-05)$ & $4,10(\mathrm{E}-05)$ & $1,30(E-05)$ & $9,00(\mathrm{E}-06)$ & 2,20 (E-05) \\
\hline
\end{tabular}

Fuente: elaboración de los autores.

Nota: el caso base se calculó usando una razón incidencia/mortalidad de 35\%, con un límite inferior de $20 \%$ y un límite superior de $50 \%$, conforme a los valores identificados en la literatura y los encontrados para el país en el presente estudio. En las mujeres, se estimó que el riesgo de desarrollar tétanos fue bajo o nulo en algunas edades debido a la menor probabilidad de la enfermedad por la vacunación en edad fértil y en estado de embarazo.

pone nulo, dada la baja probabilidad de que un individuo sufra dos episodios de tétanos de manera independiente.

La evaluación tiene en cuenta el sexo como una variable asociada a las probabilidades de desarrollar tétanos y de morir por otras causas. La discriminación por sexo se debe a que las mujeres embarazadas reciben esta vacuna y por lo tanto su riesgo de infección es menor que en los hombres. El modelo supone que la cobertura de vacunación se mantiene constante a través del tiempo. Las probabilidades de desarrollar infección por tétanos (cuadro 2) fueron estimadas a partir de los datos de mortalidad para el período 2002-2006, aplicando la siguiente fórmula, donde la incidencia (I):

$$
\mathrm{I}=\frac{\begin{array}{c}
\text { Mortalidad por tétanos } \\
\text { (reportada por el DANE) }
\end{array}}{\begin{array}{c}
\text { Razón mortalidad/incidencia } \\
\text { (reportada en la literatura) }
\end{array}}
$$

Las estimaciones de incidencia se realizaron por sexo y grupos de edad en quinquenios, mientras que las probabilidades de morir por otras causas fueron 
estimadas a partir de datos del DANE. Para el cálculo de las distribuciones de probabilidad usadas en los análisis de sensibilidad probabilísticos se supuso que las probabilidades seguían una distribución beta y los costos una distribución lognormal. El uso de beta para probabilidades está justificado ya que es una distribución acotada entre cero y que uno, lo cual garantiza que las probabilidades nunca van a ser mayores que uno ni menores que cero. En cuanto a la distribución lognormal para los costos, su uso se sustenta en que siempre toma valores positivos, garantizando así que los costos siempre sean positivos, y contempla la posibilidad de costos extremos. Para el cálculo de los parámetros de las distribuciones se supuso un error estándar de $20 \%$ de la media. El cálculo de los parámetros se muestra a continuación.

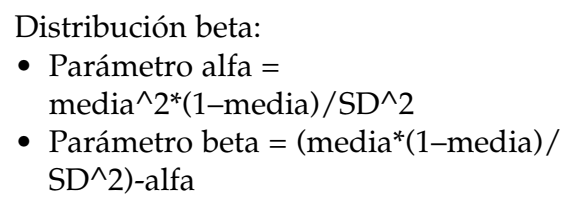

Distribución LogNormal

- Mediana = EXP(Ln(media) $\left.\operatorname{Ln}\left(\left(\mathrm{SD}^{\wedge} 2\right) / \operatorname{media}^{\wedge} 2\right)+1\right) / 2$

- $\mu=\operatorname{Ln}($ mediana)

- $\sigma=\left(2^{*} \operatorname{Ln}(\text { media } / \text { mediana })\right)^{\wedge} 0,5$

Costos de atención. El análisis de costos se realizó con la técnica de costeo del caso tipo, mediante entrevistas a profesionales de la salud con experiencia en esta clase de pacientes, quienes respondieron a un cuestionario no estructurado de siete preguntas que valoraban el uso de recursos humanos y medios diagnósticos y terapéuticos. En cada ítem se preguntó la frecuencia de uso y la dosis del tratamiento.

Los datos fueron integrados en una base de datos de MS Excel ${ }^{\circledR} 2007$. El costo de hospitalización en sala general y en unidad de cuidado intensivo (UCI) fue calculado a partir de precios de atención a particulares en una UCI y un hospital de Cartagena de Indias, Bolívar. Los precios de los medicamentos fueron tomados del comercio, en tanto que los precios de exámenes de laboratorio correspondieron al manual tarifario del Seguro Obligatorio de Accidentes de Tránsito (SOAT) 2008 para Colombia.

La estimación de costos más alta se usó como límite superior, la más baja como límite inferior y el promedio como el mejor estimado de los costos de un caso de tétanos en Colombia. El estudio tuvo la perspectiva del tercer pagador $\mathrm{y}$ solo se incluyeron costos directos de atención. Los costos se expresan en dólares estadounidenses de 2007, con un tipo de cambio de COP\$ 2078 por dólar (27).

Razón de costo-efectividad incremental. Como medida de desenlace se utilizaron los años de vida ajustados por discapacidad (AVAD), los cuales se calculan como la suma de los años perdidos por muerte prematura más los años perdidos por discapacidad. Para el cálculo de los años perdidos por discapacidad se usaron los pesos informados por la Organización Mundial de la Salud (OMS) (26).

La razón de costo-efectividad incremental (RCEI), definida como el cociente entre la diferencia de costos y la diferencia de efectos, revela cuánto cuesta un AVAD evitado al pasarse de una estrategia a otra más efectiva.

$$
\begin{aligned}
& \text { Costos incrementales con } \\
& \text { la vacunación - } \\
& \text { RCEI }=\frac{\text { Costos evitados de tratamiento }}{\text { AVAD con refuerzo }-} \\
& \text { AVAD sin refuerzo }
\end{aligned}
$$

Como lo recomienda la OMS, se consideró que una estrategia es muy costoefectiva si el costo por AVAD evitado está por debajo del producto interno bruto (PIB) per cápita, costo-efectiva si está entre 1 y 3 veces el PIB per cápita y no costo-efectiva si es mayor a 3 veces el PIB per cápita. En Colombia el PIB per cápita en 2007 fue de US\$ $4903(28,29)$.

Análisis de sensibilidad. Se realizó un análisis de sensibilidad determinístico sobre los costos, la efectividad de la vacuna, el riesgo de infección y los supuestos del modelo. Por otro lado, se hizo un análisis de sensibilidad probabilístico y se construyeron regiones de confianza y curvas de aceptabilidad.

\section{RESULTADOS}

Las tres fuentes de información para el número de casos y de muertes presentaron discrepancias (cuadro 3). El SIVIGILA (2000-2009) reportó una media de 47,9 casos por año y los RIPS (2003-2007) una media de 30 casos por año, con una razón SIVIGILA/RIPS de 3:2. En el análisis por años, ambas fuentes concordaron en un pico de incidencia para 2005: 0,19 $\times$ 100000 habitantes por SIVIGILA y $0,15 \times$
100000 por RIPS. Posteriormente, las dos registraron un descenso en la incidencia que va hasta $0,09 \times 100000$ habitantes en SIVIGILA y 0,03 × 100000 en RIPS.

Los mayores de 65 años fueron el grupo de edad con más alto riesgo en $2005\left(0,70 \times 10^{5}\right.$ por RIPS y 2,25 casos $\times$ $10^{5}$ por SIVIGILA). El registro de estadísticas vitales del DANE reportó 48 muertes por TNN en 17 departamentos (tasas de incidencia entre 0,05 y 1,24 $\times 10^{5}$ ), mientras que la letalidad por tétanos en algunos departamentos (Bolívar, Sucre y Atlántico) estuvo entre 9,28\% y 13,40\%.

\section{Análisis económico}

El modelo estimó que el riesgo de morir por tétanos es de $0,013 \%$ y $0,006 \%$ en hombres y mujeres, respectivamente, observando además que los picos de incidencia para ambos sexos ocurrieron en adultos mayores. Calculó asimismo que en promedio anualmente ocurren 70 casos de tétanos en hombres y 30 en mujeres. En la figura 2 se muestra el riesgo acumulado de morir por tétanos y en la figura 3, el número de casos incidentes por 100000 habitantes por sexo y edad.

En el cuadro 4 se observan las muertes, casos y AVAD evitados con el refuerzo y los costos asociados a la carga de enfermedad en los diferentes escenarios. Sin diferenciar por sexo, el costo por caso evitado, muerte evitada y AVAD evitado fue de US\$ 8 865, US\$ 26595 y US\$ 11314, respectivamente. Con este resultado, la RCEI de la vacunación con refuerzo a toda la población resultó ser costo-efectiva. Sin embargo, si los números se desagregan por género, en hombres el costo por AVAD evitado fue de US\$ 4903 , es decir que la vacunación fue costo efectiva, mientras que en las mujeres fue de US\$ 22 332, es decir no costo-efectiva.

\section{Análisis de sensibilidad}

Previo al análisis de sensibilidad probabilístico se realizaron análisis de sensibilidad univariados donde se observó que las RCEI son sensibles a las variaciones de la tasa de descuento, los costos de la vacuna, el riesgo de muerte por tétanos y las coberturas de vacunación.

En las figuras 4 y 5 se muestran los resultados del análisis de sensibilidad probabilístico. Para toda la población, la estrategia de vacunación más refuerzo cada 
CUADRO 3. Variaciones en los datos sobre morbilidad y mortalidad por tétanos según las fuentes oficiales de notificación, Colombia, varios años

\begin{tabular}{llcccr}
\hline & \multicolumn{1}{c}{ Fuente/Indicador } & \multicolumn{4}{c}{ Tétanos } \\
\cline { 3 - 6 } & & Accidental & Neonatal & Otros & Total \\
\hline SIVIGILA (2000-2009) & Morbilidad (casos/año) & 36,3 & 5,3 & 6,3 & 47,9 \\
& Mortalidad (casos/año) & 7,9 & 1,7 & $\ldots$ & 9,6 \\
& Grupo de edad más susceptible (años) & $\ldots$ & $\ldots$ & $\ldots$ & $>65$ \\
\multirow{2}{*}{ RIPS (2003-2007) } & Morbilidad (casos/año) & $\ldots$ & $\ldots$ & $\ldots$ & 30,0 \\
& Morbilidad (hospitalizaciones/año) & $\ldots$ & $\ldots$ & $\ldots$ & 17,8 \\
\multirow{2}{*}{ DANE (1997-2005) } & Grupo de edad más susceptible (años) & $\ldots$ & $\ldots$ & $\ldots$ & $>65$ \\
& Mortalidad (casos/año) & $\ldots$ & 5,3 & 4,8 & 10,1 \\
\hline
\end{tabular}

Fuente: elaboración de los autores a partir de las fuentes mencionadas.

Nota: Abreviaturas. SIVIGILA: Sistema de Vigilancia Epidemiológica; RIPS: Registros Individuales de Prestación de Servicios DANE: Departamento Administrativo Nacional de Estadísticas; (. . .): sin datos.

FIGURA 2. Riesgo acumulado de morir por tétanos, casos simulados a partir del modelo del presente estudio, por sexo y edad, Colombia, 2009

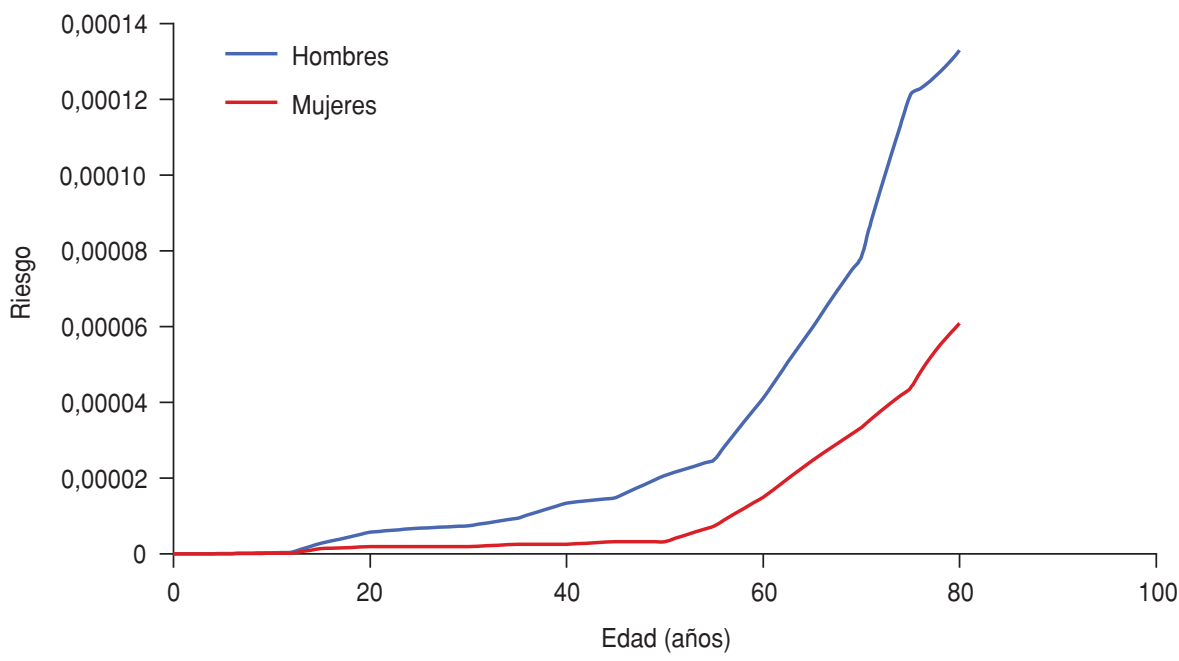

Fuente: elaboración de los autores.

FIGURA 3. Incidencia (por 100000 habitantes) de infección por tétanos estimada a partir del modelo del presente estudio, Colombia, 2009

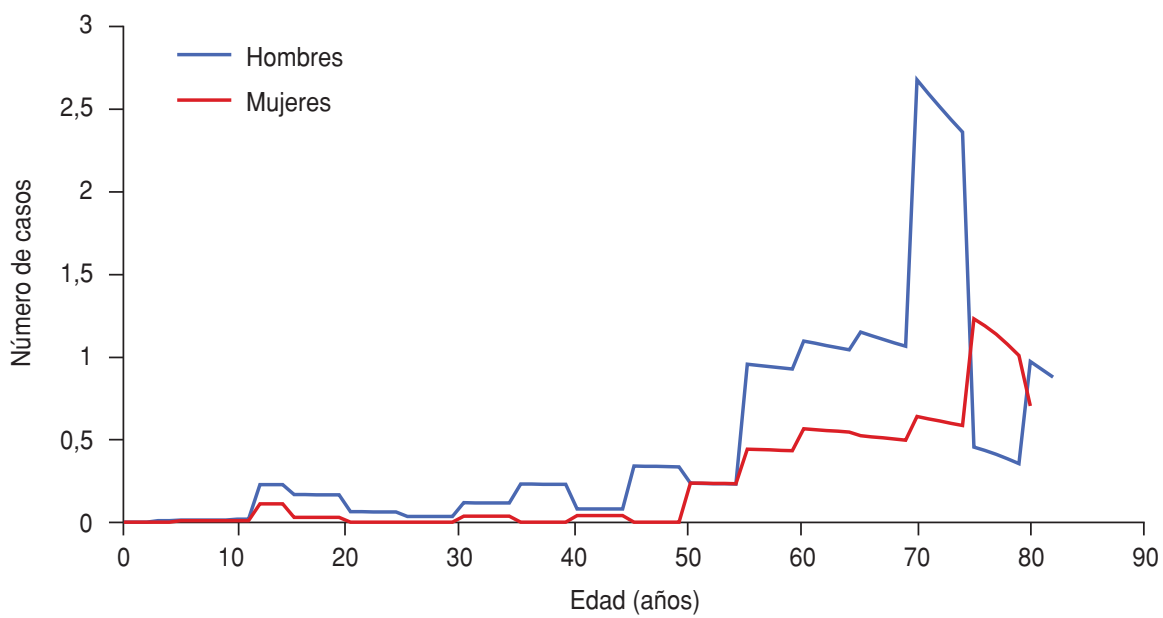

Fuente: elaboración de los autores.
10 años fue costo-efectiva si la disponibilidad a pagar fue superior a US\$13 474 por AVAD evitado. Como se puede ver en la figura 5, el área bajo la línea definida por el umbral a pagar (PIB per cápita) de la región de confianza determina la probabilidad de que la intervención sea costoefectiva, que en este caso fue de $21 \%$.

\section{DISCUSIÓN}

El presente estudio es el primero que evalúa la costo-efectividad de una dosis de refuerzo cada 10 años de la vacuna contra el tétanos en un país en desarrollo. Sus resultados revelaron que la vacunación contra el tétanos con refuerzos cada 10 años es costo-efectiva para la población colombiana. Sin embargo, el valor de la RCEI estuvo muy cerca del límite considerado aceptable de acuerdo al nivel del PIB per cápita del país. Adicionalmente, en los análisis de sensibilidad probabilísticos, solo en $21 \%$ de los casos la intervención fue costo-efectiva. Por otro lado, los resultados desagregados por género mostraron que este efecto se da principalmente en los hombres, por su mayor probabilidad de contraer tétanos. Sin embargo, debido a que no incluyó la reducción de TNN en la población de mujeres embarazadas, este modelo podría potencialmente subestimar la costo-efectividad en el género femenino, especialmente en las mujeres en edad fértil.

En Canadá, Hutchison y Stoddart (1988) hallaron que un programa de vacunación en adultos mayores de 65 años no fue costo-efectivo, resultado que podría explicarse por la baja carga de enfermedad en dicho grupo etario (30). Contrariamente, un estudio llevado a cabo en Estados Unidos (1993) encontró que la vacunación en adultos mayores sí fue costo-efectiva, mientras que la dosis de refuerzo decenal no lo fue. Esta última estrategia es la recomendación actual del plan de inmunizaciones de ese país $(31,32)$.

Si bien las diferentes fuentes de datos epidemiológicos en Colombia muestran alguna discordancia con respecto a la incidencia de tétanos, sus tasas concuerdan con las registradas en países similares, como Brasil, donde en un período de 20 años estas tasas han fluctuado entre 0,32 y 1,8 por 100000 habitantes, con tendencia al descenso (24). Mientras tanto, al comparar los resultados con los informados en países desarrollados, las tasas 
CUADRO 4. Carga de enfermedad - sin y con refuerzo- y costo-efectividad incremental de la vacunación contra el tétanos para una cohorte de 858000 nacidos vivos, Colombia, 2009

\begin{tabular}{|c|c|c|c|}
\hline \multirow[b]{2}{*}{ Escenario/Costos } & \multicolumn{3}{|c|}{ Carga de enfermedad } \\
\hline & $\begin{array}{l}\text { Base } \\
\text { (No.) }\end{array}$ & $\begin{array}{l}\text { Límite inferior } \\
\text { (No.) }\end{array}$ & $\begin{array}{l}\text { Límite superior } \\
\text { (No.) }\end{array}$ \\
\hline \multicolumn{4}{|l|}{ Escenario sin refuerzo } \\
\hline Casos de tétanos & 236 & 167 & 412 \\
\hline Muertes & 81 & 30 & 206 \\
\hline$A V A D^{a}$ & 1481508 & 1481388 & 1481851 \\
\hline \multicolumn{4}{|l|}{ Escenario con refuerzo } \\
\hline Casos de tétanos & 21 & 17 & 69 \\
\hline Muertes & 13 & 8 & 13 \\
\hline AVAD & 1481337 & 1481324 & 1481358 \\
\hline \multicolumn{4}{|l|}{ Resultados } \\
\hline Casos evitados & 219 & 98 & 391 \\
\hline Muertes evitadas & 73 & 17 & 193 \\
\hline \multirow[t]{3}{*}{ AVAD evitados } & 171 & 64 & 493 \\
\hline & \multicolumn{3}{|c|}{ Costos y costo-efectividad de los escenarios } \\
\hline & (US\$) & (US\$) & (US\$) \\
\hline \multicolumn{4}{|l|}{ Costos en el escenario sin refuerzo } \\
\hline Tratamiento & 922226,1 & 922226,1 & 1916771,2 \\
\hline Vacunación & 3195203,6 & 3195203,6 & 6592313,8 \\
\hline Total & 4117429,7 & 4117429,7 & 8509084,9 \\
\hline \multicolumn{4}{|l|}{ Costos en el escenario con refuerzo } \\
\hline Tratamiento & 112225,4 & 85923,9 & 284424,1 \\
\hline Vacunación & 5972968,2 & 2249917,1 & 12213906,6 \\
\hline Total & 6058892,1 & 2534341,2 & 12326132,1 \\
\hline \multicolumn{4}{|l|}{ Costos evitados por la vacunación } \\
\hline Tratamiento & 836302,3 & 153597,7 & 1804545,8 \\
\hline Costos netos (Escenario con refuerzo - & & & \\
\hline Escenario sin refuerzo) & 1941462,4 & 881968,7 & 3817047,1 \\
\hline \multicolumn{4}{|l|}{ Razón de costo-efectividad incremental } \\
\hline US $\$ /$ Casos evitados de ambos sexos & 8865,1 & 8696,5 & 9762,3 \\
\hline US $\$$ /Muertes evitadas de ambos sexos & 26595,4 & 19777,4 & 51880,5 \\
\hline US $\$ / A V A D$ evitados de ambos sexos & 11314,0 & 7737,0 & 13706,0 \\
\hline US\$/AVAD evitados hombres & 4903,0 & 3352,8 & 5939,4 \\
\hline US $\$ / A V A D$ evitados mujeres & 22332,0 & 15271,7 & 27053,2 \\
\hline
\end{tabular}

Fuente: elaboración de los autores.

Nota: el escenario con refuerzo es el comparador que evalúa un refuerzo cada 10 años de la vacuna contra el tétanos. El escenario sin refuerzo es el que se da actualmente en Colombia, donde no hay un refuerzo cada 10 años de esta vacuna. $\mathrm{E}$ escenario base se estimó con los parámetros base de las variables, y los límites inferior y superior son análisis de escenario del mejor y peor valor de cada parámetro.

a AVAD: años de vida ajustados por discapacidad.

FIGURA 4. Análisis de sensibilidad probabilístico (curva de confianza) para analizar la costoefectividad de la vacunación contra tétanos, Colombia, 2009

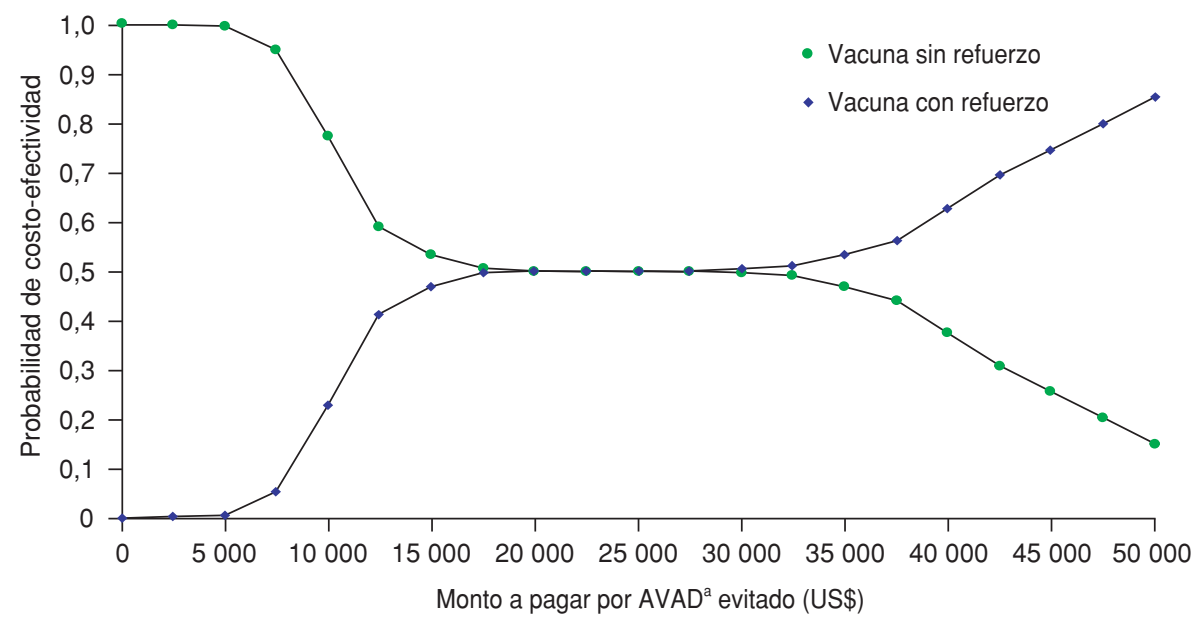

Fuente: elaboración de los autores.

a AVAD: años de vida ajustados por discapacidad. de incidencia de tétanos observadas en el presente estudio coinciden con las encontradas en 1990 en Italia, país que registró un mayor número de casos que los demás países europeos y que Estados Unidos entre 1995 y 1997 (12, 33).

En cuanto a la letalidad del tétanos accidental en Colombia, las tasas varían según los departamentos, particularmente en la costa atlántica, donde oscilan entre $30 \%$ y $100 \%$, sugiriendo probables diferencias en la calidad de la atención o en el acceso a unidades de cuidados intensivos a través del país. Comparando con Brasil, donde los resultados de estudios sobre letalidad causada por tétanos difieren de los reportados en Colombia (p. ej. tasas más elevadas en las mujeres), también hay coincidencia en que las tasas son más altas en personas mayores de 50 años de edad $(24,34,35)$. Asimismo, estudios realizados en Nigeria también se alinean con la letalidad observada entre 2000 y 2007 en algunos departamentos de la costa atlántica colombiana (30\% al $50 \%)$ $(36,37)$. En este sentido, en Colombia la letalidad es más alta (superior a 43\%) en departamentos con mayor población rural o con poco acceso a servicios de salud adecuados, como son Amazonas, Caquetá, Guaviare y La Guajira.

Cabe señalar que la divergencia entre diferentes fuentes de datos y la posible subestimación del impacto en las mujeres constituyen una limitación importante del estudio. Es probable que los datos del SIVIGILA sean los más confiables, debido a que el tétanos es una enfermedad de notificación obligatoria y este organismo realiza investigaciones de campo de los casos, a diferencia de los RIPS, que son registros rutinarios de consulta o de hospitalización donde la calidad del diagnóstico no es evaluada ni ajustada. Adicionalmente, los RIPS no están disponibles para análisis en su totalidad porque alrededor de $40 \%$ no superan el filtro de calidad de información que impone el Ministerio de la Protección Social, por lo que solo se pueden utilizar entre $40 \%$ y $50 \%$ del total.

Aun cuando también es probable que exista una subestimación del efecto de la vacunación en las mujeres, la diferencia no debería ser muy importante. La principal razón de no haber encontrado costo-efectiva la aplicación de refuerzos en las mujeres es el hecho de que la mortalidad por tétanos accidental es mucho mayor en hombres. Además por ser un 
FIGURA 5. Análisis de sensibilidad probabilístico (área de confianza) para analizar la costoefectividad de la vacunación contra tétanos, Colombia, 2009

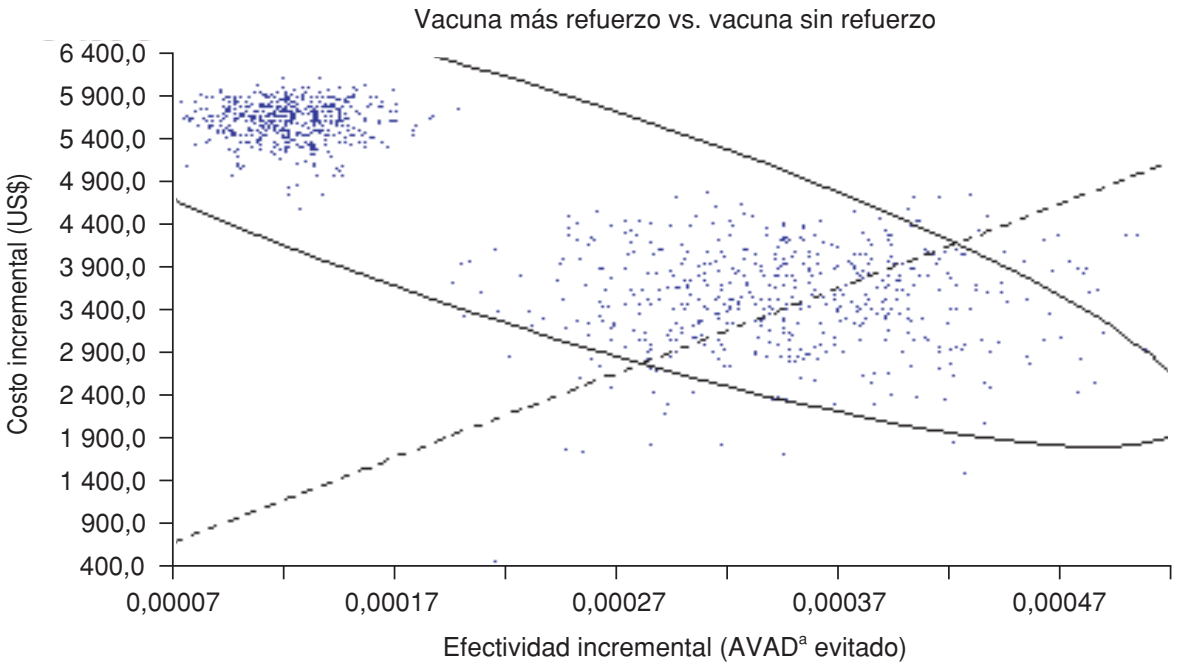

Fuente: elaboración de los autores.

a AVAD: años de vida ajustados por discapacidad.

país que está eliminando el TNN, en Colombia las mujeres reciben múltiples dosis de toxoide tetánico durante el embarazo y la inmunidad resultante de esta exposición frecuente a la vacunación bien podría ser la causa de que se presenten muchos menos casos en mujeres que en hombres. Más todavía, la vacunación frecuente durante los años de vida fértil podría hacer innecesario el re- fuerzo de tétanos en mujeres antes de los 60 años.

Por último, en la revisión de la literatura se encontró que la mortalidad infantil general en "no vacunados" es significativamente más alta en niños que en niñas, sugiriendo entonces que la reducción de la mortalidad por tétanos en hombres es mucho mayor (38). Estas consideraciones son claves para la toma de cualquier decisión de introducción de nuevos refuerzos en la vacunación contra el tétanos.

En conclusión, la aplicación de refuerzos de tétanos en adultos en Colombia es costo-efectiva, pero en un nivel cercano al umbral de no serlo debido a que el valor de la RCEI es más de dos veces el PIB per cápita. Por lo tanto, la decisión de introducir esta intervención debe tomarse teniendo en cuenta la posible sobrecarga de actividades que puede traer aparejadas y su impacto presupuestal en el programa ampliado de inmunizaciones. Adicionalmente, los beneficios netos potenciales de tal intervención - las muertes prevenidas - deben ser comparados con los producidos por otras vacunas disponibles.

\section{REFERENCIAS}

1. Cherry JD, Harrison RE. Tetanus. In: Feigin RD, Cherry JD, Demmler GJ, Kaplan SL, eds. Textbook of Pediatric Infectious Diseases. 5th ed. Philadelphia: W. B. Saunders; 2003. Pp. 1766-76.

2. Farrar JJ, Yen LM, Cook T, Fairweather N, Binh N, Parry J, et al. Tetanus. J Neurol Neurosurg Psychiatry. 2000;69(3):292-301.

3. Pendino JC, Solera JJ. Tétanos y botulismo. Medicine. 2010;10(51):3410-7. Disponible en: http:/ / www.elsevier.es/es/revistas / medicine-62/tetanos-botulismo-13148678actualizaciones-enfermedades-infecciosas2010 Acceso el 29 de mayo de 2011.

4. Centers for Disease Control and Prevention. Human Rabies Prevention - United States, 2008. Recommendations of the Advisory Committee on Immunization Practices. MMWR Recomm Rep. 2008;57(RR-3):1-28.

5. Ministerio de la Protección Social, Colombia. Esquema Nacional de Vacunación. Bogotá: Ministerio de la Protección Social; 2010.

6. Brett EM. Paediatric Neurology. 3. ${ }^{\mathrm{a}}$ ed. Londres: Churchill Livingstone; 1997.

7. Arango Soto D, Betancur Franco LA, Aguirre Muñoz C, Quevedo Vélez A. Tétanos: Todavía un problema de salud pública. Iatreia. 2008;21(2):186-98.

8. Ministerio de la Protección Social (MPS), Colombia; Organización Panamericana de la Salud. Enfermedades inmunoprevenibles en Colombia, 1997-2000. Boletín Epidemiológico. Bogotá: OPS/MPS; 2000.
9. Ministerio de la Protección Social (MPS), Colombia; Organización Panamericana de la Salud. Informe semestral. Enfermedades de notificación obligatoria. Boletín Epidemiológico. Bogotá: OPS/MPS; 2003.

10. Bleck TP. Clostridium tetani (Tetanus). In: Mandell GL, Bennett JE, Dolin R, eds. Bennett's Principles and Practice of Infectious Diseases. 6th ed. Philadelphia: Churchill Livingstone; 2000. Pp. 456-535.

11. Pascual FB, McGinley EL, Zanardi LR Cortese MM, Murphy TV. Tetanus surveillance - United States, 1998-2000. MMWR Surveill Summ. 2003;52(3):1-8.

12. Pedalino B, Cotter B, Ciofi degli Atti M, Mandolini D, Parroccini S, Salmaso S. Epidemiology of tetanus in Italy in years 19712000. Euro Surveill. 2002;7(7):103-10.

13. Cook TM, Protheroe RT, Handel JM. Tetanus: a review of the literature. Br J Anaesth. 2001; 87(3):477-87.

14. Health Protection Agency. Tetanus: information for health professionals, 2003. Disponible en: http://www.hpa.org.uk/infections/topics az/tetanus/tetanus_health_professionals.pdf Acceso el 11 de junio de 2011.

15. Whitman C, Belgharbi L, Gasse F, Torel C, Mattei V, Zoffmann H. Progress towards the global elimination of neonatal tetanus. World Health Stat Q. 1992;45(2-3):248-56.

16. Organización Panamericana de la Salud, Organización Mundial de la Salud. 128. ${ }^{a}$ Sesión del Comité Ejecutivo. Vacunas e inmuni- zación. Washington, D.C.: OPS; 2001. Disponible en: http://www.paho.org/spanish/ gov/ce/ce128_r8-s.pdf Acceso el 11 de junio de 2011.

17. Organización Panamericana de la Salud, Organización Mundial de la Salud. Informe de la XV reunión del Grupo Asesor Técnico Sobre Enfermedades Prevenibles por Vacunación. Washington, D.C.: OPS; 2002. Disponible en: http://www.paho.org/Spanish/HVP/HVI/ tag15_conclusions.pdf Acceso el 12 de junio de 2011.

18. Forest B, Castillo J, Vandelaer J, Vicari A. Evaluación del Plan Nacional de Eliminación del Tétanos Neonatal de Colombia. Bogotá: Organización Panamericana de la Salud/Ministerio de la Protección Social; 2009.

19. Arana A, Aguirre C. Tétanos en pediatría: Actualización y presentación de casos. Actualizaciones Pediátricas. 1997;7:52-60.

20. Castillo JO. Vigilancia de tétanos. Colombia. Informe Quincenal Epidemiológico Nacional. 2006;11:281-96

21. Dirección Seccional de Salud de Antioquia Colombia; Organización Panamericana de la Salud. Eventos de interés en salud pública por municipios, regiones - Antioquia; 2003. Situación de salud en Antioquia, Indicadores básicos. Medellín: Secretaría Departamental de Salud de Antioquia; 2003.

22. Escobar A, Blanco I, Velasco A, Tous F Sequeda J, Parra E. Tétanos neonatal en Car- 
tagena ¿Existen control y profilaxis? Experiencia con 196 casos. Pediatría (Bogotá). 1999; 34(2):92-9.

23. Ministerio de la Protección Social (MPS), Instituto Nacional de Salud, Colombia. Protocolo de vigilancia de tétanos accidental. Bogotá: MPS; 2008. Disponible en: http:// www.saludcordoba.gov.co/archivos_de descarga/protocolo/inmunoprevenibles/ tetanosaccidentalf.pdf Acceso el 12 de junio de 2011.

24. Gouveia PA, Silva CE, Miranda Filho Dde B, Bernardino SN, Escariao AG, Ximenes RA. Mortality trend due to accidental tetanus from 1981 to 2004 in Pernambuco and analysis of the impact on intensive care unit attendance. Rev Soc Bras Med Trop. 2009; 42(1):54-7.

25. Trujillo M. Tétanos. Manejo intensivo, complicaciones y mortalidad. Med Crit Venez. 1987;2(4):89-96.

26. World Health Organization. Global burden of disease 2004 update: disability weights for diseases and conditions. Geneva: WHO; 2004.

27. Banco de la República, Colombia. Producto interno bruto total y por habitante. Bogotá: Banco de la República; 2009. Disponible en: http:// www.banrep.gov.co/series-estadisticas/ see_prod_salar_200.htm Acceso el 12 de junio de 2010.

28. World Health Organization. Macroeconomics and health: investing in health for economic development. Geneva: World Health Organization; 2001.

29. Banco de la República, Colombia. Series Estadísticas. Producción, salarios y empleo. Bogotá: Banco de la República; 2009.

30. Hutchison BG, Stoddart GL. Cost-effectiveness of primary tetanus vaccination among elderly Canadians. CMAJ. 1988;139(12):1143-51.

31. Balestra DJ, Littenberg B. Should adult tetanus immunization be given as a single vaccination at age 65 ? A cost-effectiveness analysis. J Gen Intern Med. 1993;8(8):405-12.

32. Centers for Disease Control and Prevention. Recommended adult immunization scheduleUnited States, 2011. MMWR Morb Mortal Wkly Rep. 2011;60(4):1-4.

33. Bardenheier B, Prevots DR, Khetsuriani N, Wharton M. Tetanus surveillance - United States, 1995-1997. MMWR CDC Surveill Summ. 1998;47(2):1-13.

34. Feijao AR, de Brito DM, Peres DA, Galvao MT. Accidental tetanus in the State of Ceara, between 2002 and 2005. Rev Soc Bras Med Trop. 2007;40(4):426-30.

35. Greco JB, Tavares-Neto J, Greco Junior JB. Accidental tetanus: prognosis evaluation in a historical series at a hospital in Salvador Bahia, Brazil. Rev Inst Med Trop Sao Paulo. 2003;45(1):35-40.

36. Chukwubike OA, God'spower AE. A 10-year review of outcome of management of tetanus in adults at a Nigerian tertiary hospital. Ann Afr Med. 2009;8(3):168-72.

37. Adudu OP, Ogunrin OA, Adudu OG. Morbidity and mortality patterns among neurological patients in the intensive care unit of a tertiary health facility. Ann Afr Med. 2007;6(4):174-9.

38. Chan GJ, Moulton LH, Becker S, Munoz A, Black RE. Non-specific effects of diphtheria tetanus pertussis vaccination on child mortality in Cebu, The Philippines. Int J Epidemiol. 2007;36(5):1022-9.

Manuscrito recibido el 18 de septiembre de 2010. Aceptado para publicación, tras revisión, el 27 de abril de 2011.

Objective. Assess the potential epidemiological and economic impact of vaccinating the over-15 Colombian population against tetanus with a booster dose every 10 years.

\section{Epidemiological and economic impact of tetanus vaccination in Colombian adults}

Key words
Methods. A cost-effectiveness analysis of tetanus vaccination with a booster dose every 10 years was conducted in Colombia and compared with the current strategy $(2,4,6,18$, and 60 months). Estimates of the burden of disease were based on three official data sources. A Markov model from the perspective of the third party payer was developed. The time horizon was the lifetime of a person. Deterministic and probabilistic sensitivity analyses were conducted.

Results. In Colombia, 30 to 48 cases of tetanus resulting in 9.6 to 10.1 deaths are reported each year. Although booster vaccination for the entire population was costeffective (the cost per disability-adjusted life year [DALY] avoided was US\$ 11,314 in the entire population), gender-based differentiation of the results showed that it would not be cost-effective in women (cost per DALY avoided was US\$ 4,903 in men and US\$22,332 in women).

Conclusions. This is the first study that evaluates the cost-effectiveness of a tetanus vaccine booster dose every 10 years in a developing country. Use of this measure would be cost-effective in Colombia, especially for men. As a result of the genderbased differences in the results, any decision about its use in women of childbearing age should take current vaccination into account.

Tetanus toxoid; health economics; maternal and child health; Colombia. 